\title{
Characterization of p-type Doping in Silicon Nanocrystals Embedded in $\mathrm{SiO}_{2}$
}

\author{
R. Demoulin ${ }^{1}$, M. Roussel ${ }^{1}$, S. Duguay ${ }^{1}$, D. Muller ${ }^{2}$, D. Mathiot ${ }^{2}$, P. Pareige $^{1}$ and E. Talbot ${ }^{1, *}$. \\ 1. Normandie Univ, UNIROUEN, INSA Rouen, CNRS, Groupe de Physique des Matériaux, 76000 \\ Rouen, France \\ 2. ICube Laboratory, Université de Strasbourg and CNRS, B.P. 20, 67037 Strasbourg Cedex, France. \\ * Corresponding author: etienne.talbot@univ-rouen.fr
}

Materials consisting of silicon nanocrystals ( $\mathrm{Si}-\mathrm{Ncs})$ embedded in silicon dioxide $\left(\mathrm{SiO}_{2}\right)$ are the subject of an intense research activity due to their potential applications for optoelectronic and photonic devices. Moreover, providing charged carriers by introducing $n$ - or $\mathrm{p}$-type dopants in these materials can drastically modify their electrical or optical properties. A plenty of studies focused on the capabilities to change the indirect bandgap of Si-Ncs to direct one and to improve the luminescence efficiency of SiNcs by single or co-doping. Recently a particular interest was focused on the localized surface plasmon resonance exhibited by highly doped Si-Ncs [1]. In both cases, a perfect control of the doping level and size of Si-Ncs should allow to tune the material properties. However, efficiency of these doped materials is correlated to the dopant location, which should be located in a substitutional site of the Si-Ncs. Then, it requires an accurate control of this parameter to improve and control the properties of these systems. Numerous studies concern the characterization of P doping in Si-Ncs, here, we propose to compare undoped and p-doped (with P or As) Si-Ncs by the use of Atom Probe Tomography (APT) to perform a deep structural analysis at the atomic scale. Further investigations will be done on n-type (B) doping.

Three silicon rich silicon oxide films, undoped and As or P doped, were elaborated by using ion beam synthesis process. Implantation was divided into two parts. First one consists in a ${ }^{29} \mathrm{Si}$ implantation in a ${ }^{28} \mathrm{SiO}_{2} 200 \mathrm{~nm}$ thick layer. Second one consists, for two samples, in another implantation of ${ }^{75} \mathrm{As}$ or ${ }^{31} \mathrm{P}$ chose to match with the ${ }^{29} \mathrm{Si}$ implantation range. Finally, samples were annealed at $1100^{\circ} \mathrm{C}$ during $4 \mathrm{~h}$ in pure $\mathrm{N}_{2}$ to form Si-Ncs. Structural characterization of these thin films was performed combining Timeof-Flight Secondary Ion Mass Spectrometry (Tof-SIMS) and Atom Probe Tomography. Measurements performed in Tof-SIMS allowed us to ensure the reliability of 3-D reconstruction of the tip samples.

In undoped as in As or P doped samples, Tof-SIMS revealed a region of interest, corresponding to the implantation range of ${ }^{29} \mathrm{Si}$ in the ${ }^{28} \mathrm{SiO}_{2}$ thin films, of almost $110 \mathrm{~nm}$ of thickness and centered at barely $55 \mathrm{~nm}$ from the film surface. Moreover, for As or P doping, it confirms that the implantation of ${ }^{29} \mathrm{Si}$ and both dopants species match well in the same region. We must note that considering APT composition profiles, the composition reached at the peak center of the implantation by ${ }^{29} \mathrm{Si}(\sim 11$ at.\%) and As or P $(\sim 1.2$ at.\%) in each sample is almost the same. In each case, $3-\mathrm{D}$ chemical maps obtained after the APT reconstruction show that the annealing treatment performed at $1100^{\circ} \mathrm{C}$ leads to the clustering of ${ }^{29} \mathrm{Si}$ and

${ }^{28} \mathrm{Si}$ without any distinction. The mapping of As and $\mathrm{P}$ atoms allowed us to highlight the aggregation of the impurities at the same position than the clustering of Si atoms. Thereby, from a first global view, both doping cases seems to be equivalent. The use of APT allowed us to perform deeper investigation on the Si-Ncs characteristics and on the dopant location. In all samples, the diameter of Si-Ncs has been measured in order to compare the size distributions of Si-Ncs. In undoped as in As doped samples, mean diameter and shape of size distribution are almost the same, but we observed strong difference of these parameters in $\mathrm{P}$ doped sample (figure 1.a, 1.b). In fact, for undoped and As doped samples, size 
distributions show a lognormal shape with a mean diameter of $2.5 \pm 0.4 \mathrm{~nm}$ whereas for P doped sample it shows a Gaussian shape with bigger diameters $(\sim 3.3 \pm 1.0 \mathrm{~nm})$. Moreover, an investigation of these size distributions along the implantation profile highlighted a similar evolution of Si-Ncs diameter between undoped and As doped thin layers (figure 1.c). It allowed us to conclude that introduction of As atoms does not impact the Si-Ncs growth process whereas $\mathrm{P}$ atoms have strong influence on it. This may be attributed to a change of the Si diffusion length of Si atoms in presence of $\mathrm{P}$ atoms [2]. From a doping point of view, it has been shown that all Si-Ncs contain P or As atoms (figure 2.a). The computation of erosion profiles, which represent the evolution of species composition from the center of the Si-Ncs to the surrounding matrix, allowed us to conclude about an efficient introduction of impurities (As or P) in the core of the Si-Ncs without any pile-up of impurities at the $\mathrm{Si} / \mathrm{SiO}_{2}$ interfaces (figure 2.b), even for the smaller Si-Ncs for which self-purification effect could occur [3]. The impurity composition of each $\mathrm{Si}-\mathrm{Ncs}$ have been computed. Its representation as a function of the Si-Nc diameter shows that both impurities follow the same tendency in term of impurity level in the Si-Ncs (figure 2.c). On one side, bigger Si-Ncs (above $3 \mathrm{~nm}$ ), tend to stabilize at an equilibrium state corresponding to an impurity level of 5 at.\%. On the other side, majority of the smallest Si-Ncs (below $2 \mathrm{~nm}$ ) exhibits higher impurity level which can reaches values up to 45 at.\%. This allowed us to highlight a size dependency of the p-doping level in Si-Ncs, and show that the formation of highly p-doped small Si-Ncs may be performed.

\section{References:}

[1] J. M. Luther et al, Nature Materials 10 (2011), p. 361-366.

[2] G. M. Dalpian, J.R. Chelikowsky, Physical Review Letter 96 (2006), p. 226802.

[3] M. Fujii et al, Journal of Applied Physics 93 (2003), p. 1990-1995.
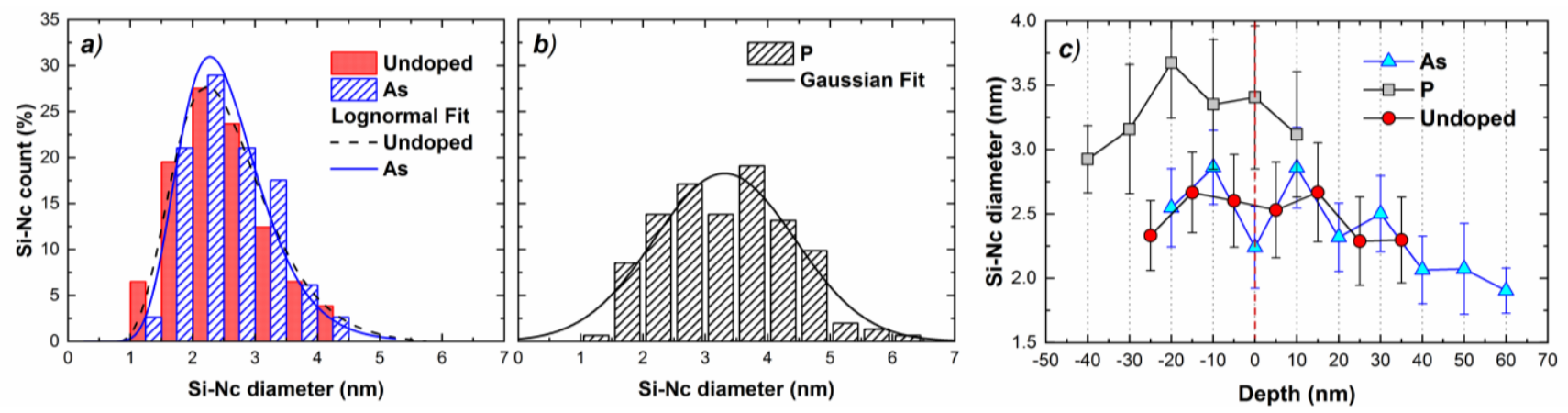

Figure 1. Size distribution of (a) undoped, As doped and (b) P doped Si-Nes. Solid and dashed lines represent distribution fitting. (c) Diameter evolution along the implantation profile.
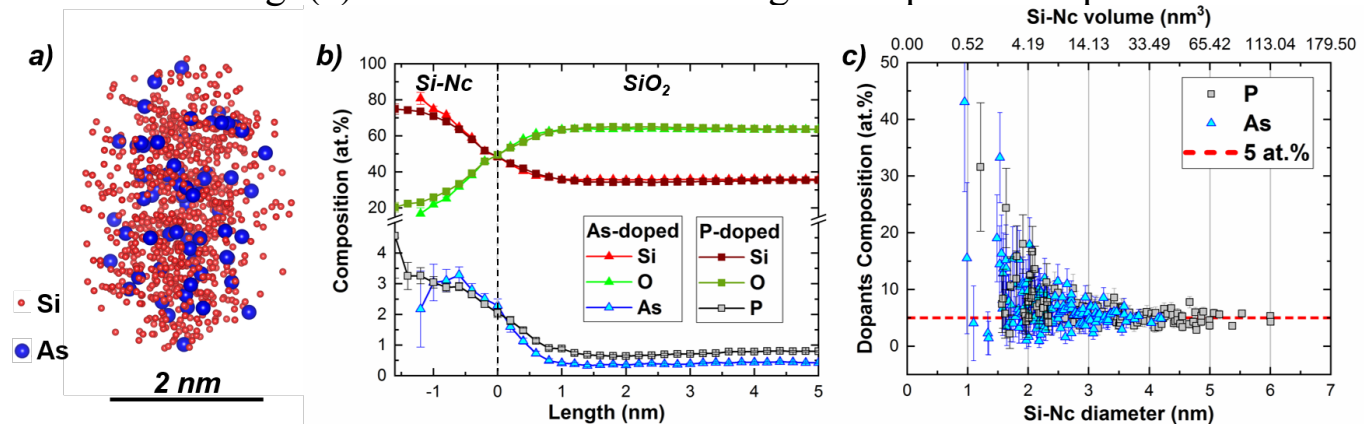

Figure 2. (a) 3-D reconstruction of As doped Si-Nc. (b) Mean erosion profile of As and P doped samples. (c) Impurity composition of each Si-Ncs as a function of their diameter. 